\title{
Binocular detection of positive and negative flashes*
}

\author{
DAVID H. WESTENDORF and ROBERT FOX \\ Vanderbilt L'niversity', Nashrille, Tennessee 37240
}

\begin{abstract}
Binocular forced-choice detection rates were measured under conditions where both eyes received positive flashes. both eyes received negative flashes, and one eye received a positive flash while the other received a negative flash. When both eyes received the same kind of nash, both positive or both negative. detection rates were greater than probability summation. When one eye received a positive flash while the other received a negative flash. detection rates were near a level expected on the basis of probability summation. It is concluded that, at the level of forced-choice detection. positive and negative flashes are detected as though they were separate, independent events.
\end{abstract}

For several threshold tasks, including increment detection, binocular performance is superior to monocular by an amount greater than that predicted by appropriate probability summation models (see review by Blake \& Fox, 1973). Superior binocular performance must, therefore. be attributable to a facilitatory neural interaction between the eyes, a phenomenon referred to as binocular summation.

In the present experiment, the presence of binocular summation was tested for the case where flashes to each eye were equally detectable, defined by a forced-choice detection criterion. but the flash to one eye was positive while to the other it was negative. Positive flashes refer to brief increases in stimulus energy. while negative flashes refer to brief decreases in energy. One reason for an interest in this stimulus combination is that most of the previous experiments on binocular summation have employed identical stimul us conditions for each eye, and it is not known if these conditions are essential for demonstrating summation. at least at the threshold level. In an earlier experiment (Westendorf, Blake, \& Fox, 1972), a difference in each eye's stimulus was introduced by varying the luminance and duration of positive flashes so that they were equally detectable in accord with Bloch's law. That is, one eye would receive a relatively bright brief flash and the other a relatively dim long flash, both flashes presented simultaneously. Flashes whose luminance/duration characteristics differed by a factor of three were as detectable under forced-choice conditions as flashes with identical luminance/duration characteristics; performance in both cases was significantly better than predicted by probability summation. These data can be interpreted as supporting a hypothesis of general energy integration, which assumes that at threshold the binocular detection system acts only on stimulus energy, ignoring stimulus features that would otherwise distinguish them.

The combination of positive and negative targets provides a more stringent test of the general energy integration hypothesis, since it is known that when such targets are seen continuously at suprathreshold levels

\footnotetext{
* Support for this research was provided by Grant EY00590 from the C.S. Public Health Service.
}

they engage in binocular rivalry. Further, the elegant experiments of Ikeda (1965) and Rashbass (1970) have shown that positive and negative flashes superimposed monocularly engage in inhibitory interactions. Moreover, the recent work of J.P. Thomas and his colleagues (e.g., Thomas \& Kerr, 1971) indicates that even at the level of forced-choice detection thresholds the detection system remains responsive to such features as target size. These considerations suggest that an inhibition hypothesis may be a plausible alternative to the energy integration hypothesis when the stimuli are positive and negative flashes. Several variations of an inhibition hypothesis might be entertained. but all would predict that binocular detection of positive and negative flashes would be at least no better than the detection level observed under monocular conditions.

Thus, the major purpose of the present experiment was to determine which of these two general hypotheses, inhibition or energy integration, would receive support when the stimuli to each eye are radically different, as in the case of positive and negative flashes. As a byproduct of the design, the experiment also provided information on the magnitude of summation of negative flashes compared with the magnitude of summation of positive flashes.

\section{METHOD}

\section{Subjects}

Two persons experienced in psychophysical observation served as Ss. One, R.F.. is an author of this paper. Both Ss had vision corrected to normal. R.F. via lenses placed in the trial frames of the apparatus and B.W. via contact lenses.

\section{Apparatus}

The basic apparatus was a large haploscope fabricated from optic bench components and rigidly mounted on a steel bench. The left and right eve-field arms were optic benches $1 \mathrm{~m}$ long: each had an auxiliary arm attached at a right angle. The eye-field arms pivoted at the viewing port under the pivot points of the eves in such a way that both interpupillary distance and convergence angle were independently adjustable. The haploscope and related apparatus were enclosed in a lightproof housing fitted with internal baffles to prevent interference from stray light. An adjustable biteboard and headrest were located at the viewing port of the housing. Also at the viewing port was a 


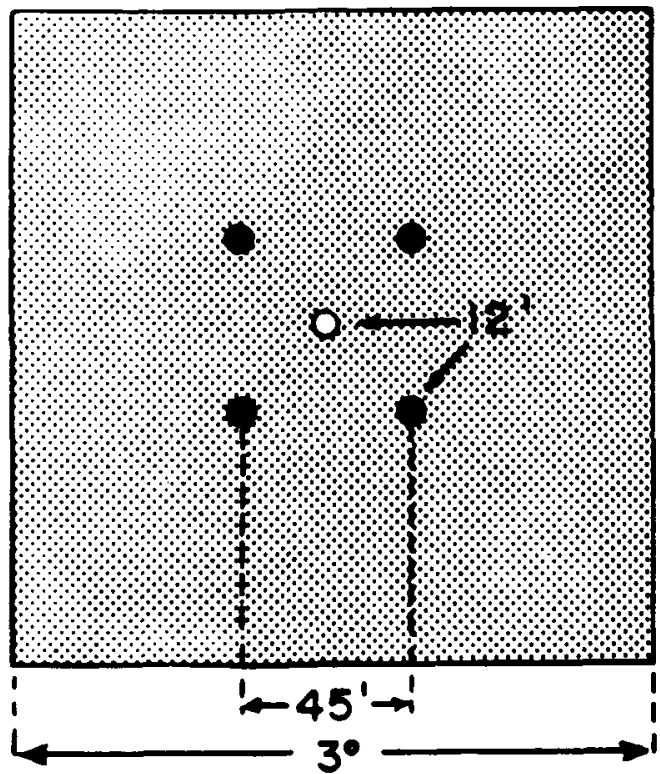

Fig. 1. Stimulus configuration: The central 12-min bright spot became momentarily brighter for positive flashes or dimmed to the surround brightness for negative flashes.

pair of trial frames suspended from an assembly that permitted vernier adjustment of the frames in three dimensions. The trial frames carried artificial pupils whose $2-\mathrm{mm}$ apertures were located in protruding conical portions so that they could be located as close as possible to the cornea.

The basic stimulus field configuration as seen by the $S$ is illustrated in Fig. 1. Four 12-min-diam black fusion markers are arranged in a 45-min square centered on a 3-deg-square white field. At the center of this field, a bright spot, $12 \mathrm{~min}$ in diam, defines the area where the test stimulus occurred. For positive flashes, the luminance of the bright central spot increased briefly; for negative flashes, the luminance of the central spot fell briefly to the background luminance level (see Fig. 2). To produce these stimulus conditions, two types of background displays were used. For positive flashes, the bright central spot was an integral part of the background display and the positive stimulus was optically superimposed on the spot. For negative flashes, the bright spot was provided by continuous presentation of the test target lamp; the brief extinction of this spot of light superimposed on the decremental background display constituted the negative stimulus. Therefore, the two types of background displays differed physically only with regard to the origin of the central spot. When a spot was superimposed on the negative background display, the resulting stimulus field configuration was phenomenally identical to the display used for positive flashes.

Background displays were fashioned from neutral density filter material mounted on translucent Plexiglas. Opaque dots on the displays were made from dry-transfer "dot rules" (Letraset). One of each type of display was placed on each arm of the haploscope in a two-position detented filter wheel, so that for any series of trials the E could easily and accurately position the wheels to display the appropriate background to each eye of the $S$. The displays were $75 \mathrm{~cm}$ from each eye of the $S$ and transilluminated by fluorescent lamps (Sylvania F15T12D). Fluorescent, rather than incandescent, lamps were used in order to provide an acceptable subjective color match between the background displays and the test lamps described below. A number of types of fluorescent bulbs were tested, and the "daylight" phosphor was found to be the most satisfactory.

Test stimuli were produced by cold-cathode mercury argon lamps placed in lamphouses mounted on the auxiliary arms of the haploscope. A collimating lens and mask located within each lamphouse were used to produce the $12-\mathrm{min}$ homogeneously illuminated spot. This spot was superimposed on the center of the background display via a beamsplitter. Test-target lamps were powered and their duration controlied by a lamp-driver unit and timing units of a three-channel tachistoscope (Scientific Prototype, Model GA).

The luminance of the 3-deg-square white patch of the background displays was $13.5 \mathrm{~cd} / \mathrm{m}^{2}$; black-spot and surround luminance was less than $.005 \mathrm{~cd} / \mathrm{m}^{2}$. The luminance of the 12-min fixation spot on the positive stimulus displays was $21.6 \mathrm{~cd} / \mathrm{m}^{2}$. Test-target luminance was $8.1 \mathrm{~cd} / \mathrm{m}^{2}$, so that when superimposed on the negative stimulus display the resultant fixation-spot luminance was $21.6 \mathrm{~cd} / \mathrm{m}^{2}$. Therefore, as illustrated in Fig. 2, at the beginning of both positive and negative flash trials, a fixation spot of $21.6 \mathrm{~cd} / \mathrm{m}^{2}$ was visible on a background of $13.5 \mathrm{~cd} / \mathrm{m}^{2}$. On positive flash trials, the fixation-spot luminance rose briefly to $29.7 \mathrm{~cd} / \mathrm{m}^{2}$; on negative trials, it fell briefly to $13.5 \mathrm{~cd} / \mathrm{m}^{2}$, the background luminance level. Fine adjustment of background display and test stimulus luminance was accomplished by placing neutral density filters behind the background displays and in the test-target lamphouses. Absolute luminance values were measured at the viewing port before each experimental session with a Pritchard Spectra spot photometer (Model 1970-PR). The luminance of the fluorescent tubes illuminating the background displays was monitored throughout experimental sessions via photocells. The waveform of the test flashes was examined using a phototube and oscilloscope. For both the left-eye and right-eye lamps, the shape of the wave was square, with rise to maximum amplitude occurring in less than $1 \mathrm{msec}$, rise to $90 \%$ amplitude occurring within $.5 \mathrm{msec}$, and decay to 0 amplitude complete within $.5 \mathrm{msec}$.

To mask various extraneous sounds, a moderate level of white noise was presented to the $S$ through headphones. To aid in securing a comfortable position while constrained by the biteboard, the $S$ sat in a motorized seat (automobile power seat) adjustable in two dimensions.

\section{Procedure}

Each trial throughout the experiment consisted of a three-choice, temporal forced-choice task with $1 / 3$ a priori probability of a correct guess by chance. Ss initiated each trial by activating a timer that controlled the following events constituting a trial. Following a $.5-\mathrm{sec}$ delay, a series of three 1 -sec tone bursts separated by $.5 \mathrm{sec}$ was presented to the $S$ via the headphones. These $1-\sec$ tones defined the observation

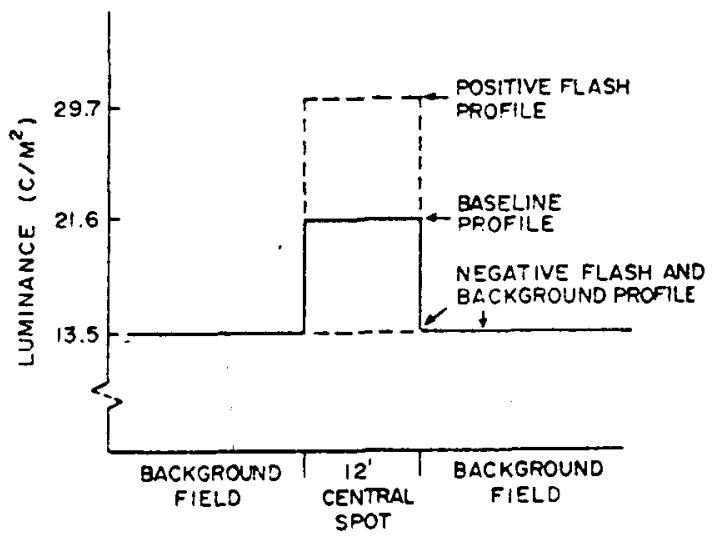

Fig. 2. Luminance profile of the central 12 -min bright spot and adjacent area together with profiles associated with positive and negative flashes. 
intervals; signal onset could occur only in the middle of each interval. Ss reported in which interval the target event had most likely occurred by pressing one of three switches. Immediately following this response, feedback was provided by a high-pitched tone, indicating a correct response, or a low-pitched tone, indicating an incorrect response. Both interval-marking and feedback signals were superimposed on the continuous white-noise background.

Prior to the experiment, each $S$ participated in a preliminary session devoted to the determination of stimulus durations that yielded about $50 \%$ correct detection rates. Modified staircase series were run under monocular conditions for each eye and for both positive and negative flashes. Four stimulus durations were used for R.F : $25 \mathrm{msec}$ for right-eye positive flashes, $15 \mathrm{msec}$ for left-eye positive flashes, $21 \mathrm{msec}$ for right-eye negative flashes, and $13 \mathrm{msec}$ for left-ey'e negative flashes. The stimulus durations used for B.W. were $25 \mathrm{msec}$ for positive flashes and $22 \mathrm{msec}$ for negative flashes. To insure that the monocular detection rates approximated the desired baseline hit rate of $50 \%$, slight adjustments of stimulus duration were made. For R.F., stimulus durations for each eye were reduced $2 \mathrm{msec}$ after the 12 th session; for B.W., stimulus durations for each eye were reduced by $1 \mathrm{msec}$ after the 4 th session and after the 8 th session.

Detection thresholds were measured under eight conditions: (1) a positive monocular condition. in which positive flashes were presented to each eye alone; (2) a positive binocular condition, in which positive flashes were presented to both eyes simul taneously; (3) a positive asynchronous condition, in which positive flashes were presented to both eyes but separated by $100 \mathrm{msec}$; (4) a negative monocular condition, in which negative flashes were presented to each eye alone; (5) a negative binocular condition, in which negative flashes were presented to both eyes simultaneously: (6) a negative asynchronous condition, in which negative flashes were presented to both eyes separated by $100 \mathrm{msec}$; (7) a mixed binocular condition, in which a positive flash was presented to one eye while a negative flash was presented to the other eye; and (8) a mixed asynchronous condition. in which a positive and a negative flash were presented, one to each eye, separated by $100 \mathrm{msec}$. In the three asynchronous conditions, a $100-\mathrm{msec}$ interval occurred between offset of the first flash and onset of the second; the onset of the first flash occurred, as in the other experimental conditions, $500 \mathrm{msec}$ after the onset of an interval-marking signal. The asynchronous conditions were included to provide an empirical baseline for assessment of probability summation. The required assumption that flashes separated by this temporal interval exhibit only probabilistic interaction is supported by Matin (1962), who found that dichoptic flash pairs separated by this interval and greater were detected at a level near that expected for the case where the two eyes are independent detectors. Westendorf, Blake, and Fox (1972) also employed asynchronous conditions. using a 100-msec delay between targets, and found performance near that predicted by two different models of probability summation. One of the $S s$ in that experiment was run under both 100 - and $200-\mathrm{msec}$ delay conditions. Performance did not differ between these two conditions.

Ss made 300 judgments for each of the eight conditions in 15 sessions. Each session consisted of eight randomized blocks of 20 trials each, with one block dedicated to each condition. Experimental conditions were not varied within blocks. For all conditions, when appropriate, type of test flash, eye of origin, and, in the case of asynchronous conditions, which eye received the furst of the two flashes were balanced across sessions. Since data were collected in 15 sessions, complete balancing of eye of origin was not accomplished. For example. the monocular performance reported below is based on 160 left-eye and 140 right-eye judgments in each monocular condition by each $\mathrm{S}$. To determine if this incomplete balance might have biased the experimental results. a subset of the data (from Sessions 1.8 of both $\mathrm{Ss}$ ) that was completely counterbalanced was examined. The pattern of results was found to be the same as that described in the next section.
Each session lasted about $45 \mathrm{~min}$. Ss always participated in at least two sessions per sitting. with about 10 min rest between sessions and briaf rest periods between blocks. No more than four sessions were run in a single day. Before any trials were run each day, at least $15 \mathrm{~min}$ was spent checking alignment of the optical system, allowing the $S$ to adapt to the lighting conditions and presenting a few practice trials. Under both monocular and binocular conditions, Ss kept both eyes open. Special attention was paid to ensure that $S \mathrm{~s}$ achieved and maintained good fusion. Ss were not informed concerning the condition being tested at any time. When queried after their last sessions, both $\mathrm{Ss}$ maintained that the only discernible difference between conditions was the relative difficulty in detecting the signal. It may be of interest to note that the question of phenomenal difference between positive and negative flashes was of special interest to R.F., yet he could not discriminate between them when hit rates were less than $100 \%$.

\section{RESULTS AND DISCUSSION}

Although all experimental conditions were run concurrently, it is helpful to examine the results in two portions. One portion concerns the performance when the flashes to both eyes were in the same direction, either both positive or both negative, and the second portion concerns the case where the flashes to the eyes were different, one positive and the other negative.

Table 1 contains the percent-correct detections for monocularly presented positive and negative flashes for each eye of each of the two Ss, detection under the binocular-summation condition for both positive flash combinations and negative flash combinations, and performance for a binocular asynchronous condition where the flashes were presented successively (separated by $100 \mathrm{msec}$ between offset of the first flash and onset of the second). Also in Table 1 are the predictions derived from two models of probability summation. ${ }^{1}$ One model is that proposed by Eriksen (1966; Eriksen. Greenspon, Lappin, \& Carlson, 1966); the specific version of the Eriksen model used for calculation is known as the two-state model and is described specifically in Eriksen et al. ${ }^{2}$ The second model, called the integration model, is derived from the theory of signal detection and is described specifically in Green and Swets (1966). ${ }^{3}$

Wilcoxon signed-ranks tests on session-by-session hit rates were used to evaluate the significance of differences in performance under the experimental conditions. For the binocular summation condition for both Ss for both positive flashes and for negative flashes. performance is significantly greater than that obtained under the binocular asynchronous condition (each $\mathrm{N}=$ $15, p<.01$ ). Since performance under both binocular summation conditions is significantly greater than that anticipated on the basis of probability summation. it is necessary to conclude that the superior performance reflects a facilitatory neural interaction. Further, there is essentially no difference between the magnitude of the summation for positive flashes relative to the summation found for negative flashes, thereby demonstrating that negative flashes have the same summation capability as the more commonly employed positive flashes. 
Table 1

Percentage of Correct Detections for Each Subject Under Each Monocular Condition, Under Binocular Conditions for Both Positive and Negative Flash Combinations, and Probability Summation Predictions of Two Probability Models

\begin{tabular}{|c|c|c|c|c|c|c|}
\hline \multirow[b]{2}{*}{$\mathrm{S}$} & \multicolumn{2}{|c|}{ Monocular } & \multicolumn{2}{|c|}{ Binocular } & \multicolumn{2}{|c|}{ Model Predictions } \\
\hline & $\begin{array}{c}\text { Positive } \\
\text { Rt/Lf }\end{array}$ & $\begin{array}{c}\text { Negative } \\
\text { Rt/Lf }\end{array}$ & $\begin{array}{l}\text { Synch } \\
\text { Pos/Neg }\end{array}$ & $\begin{array}{l}\text { Asynch } \\
\text { Pos/Neg }\end{array}$ & $\begin{array}{c}\text { Two-State } \\
\text { Pos/Neg }\end{array}$ & $\begin{array}{c}\text { Integration } \\
\text { Pos/Neg }\end{array}$ \\
\hline $\begin{array}{l}\text { R.F. } \\
\text { B.W. }\end{array}$ & $\begin{array}{l}49 / 60 \\
59 / 53\end{array}$ & $\begin{array}{l}51 / 65 \\
61 / 63\end{array}$ & $\begin{array}{l}78 / 82 \\
92 / 95\end{array}$ & $\begin{array}{l}67 / 70 \\
71 / 70\end{array}$ & $\begin{array}{l}69 / 75 \\
70 / 78 \\
\end{array}$ & $\begin{array}{l}64 / 70 \\
66 / 73 \\
\end{array}$ \\
\hline
\end{tabular}

The performance under the binocular asynchronous condition can be used as an empirical test of the adequacy of both probability summation models. It can be seen from Table 1 that there is reasonably close agreement for both Ss among the asynchronous conditions and the predictions of the two models, all agreeing with one another within a detection rate of $5 \%$. The tendency of the two-state model to overpredict relative to the integration model is to be expected, since that version of the Eriksen model does not represent all the information available to the $S$ in the detection task (see Eriksen et al for a discussion of this point). The correspondence between both models and with the asynchronous condition observed in this experiment has also been found by Westendorf, Blake, and Fox (1972).

Considering now the data for the binocular combination of positive and negative flashes, Table 2 contains the monocular detection rates for both Ss, the detection rate under the binocular summation condition, and the detection rate under the binocular asynchronous condition. Performance under the summation condition is very similar to that obtained under the asynchronous condition, and application of the Wilcoxon test reveals that these differences are not significant (each $N=15$, $p<.05$ ). In effect, the performance under the binocular summation condition when negative and positive flashes are paired is at the same level as that anticipated by probability summation. This result argues against any version of the inhibition hypothesis discussed in the introduction. Unlike the monocular case studied by Ikeda (1965) and by Rashbass (1970), dichoptically presented positive and negative flashes to not cancel one another. The absence of inhibitory interaction at the more central interocular stage of processing is consistent

Table 2

Percentage of Correct Detections for Each Subject Under Monocular Conditions (Averaged Over Positive and Negative Flashes), Under Binocular Conditions for Positive and Negative Flash Combinations, and Probability Summation Predictions of Two Probability Models

\begin{tabular}{|c|c|c|c|c|c|c|}
\hline \multirow[b]{2}{*}{$S$} & \multicolumn{2}{|c|}{ Monocular } & \multicolumn{2}{|c|}{ Binocular } & \multicolumn{2}{|c|}{$\begin{array}{c}\text { Model } \\
\text { Predictions }\end{array}$} \\
\hline & Right & Left & Synch & Asynch & $\begin{array}{l}\text { Two- } \\
\text { State }\end{array}$ & $\begin{array}{l}\text { Inte- } \\
\text { gration }\end{array}$ \\
\hline $\begin{array}{l}\text { R.F. } \\
\text { B.W. }\end{array}$ & $\begin{array}{l}50 \\
60\end{array}$ & $\begin{array}{l}63 \\
58\end{array}$ & $\begin{array}{l}70 \\
79\end{array}$ & $\begin{array}{l}73 \\
74\end{array}$ & $\begin{array}{l}72 \\
75\end{array}$ & $\begin{array}{l}66 \\
69\end{array}$ \\
\hline
\end{tabular}

with the interpretations of Ikeda and of Rashbass, that positive and negative monocular flash interaction is attributable to superposition of the positive and negative flash impulse functions occurring within the peripheral receptor. The present data suggest that a different kind of processing mode is operative at the interocular interaction stage.

The general energy integration hypothesis, described in the introduction, represents an alternative processing mode. The data, however, do not support that hypothesis, since it necessarily predicts that the positive-negative flash pairs would exhibit an equivalent amount of summation as the positive flash pairs and the negative flash pairs. Clearly, this did not occur. It should be noted that rejection of the integration hypothesis carries with it the implication that the differences between monocular stimuli are not ignored by the binocular detection system, even at the level of forced-choice thresholds.

One interpretation of this sensitivity to stimulus difference, suggested by the close correspondence of positive-negative flash pair detection performance to the level anticipated by probability summation, is that when the stimuli to each eye differ sharply, the visual system classifies the stimuli as separate independent events even though they are superimposed in space and time. The effect of this classification would be to permit only probabilistic interaction between the stimuli. This interpretation of positive-negative flash-pair performance implies that other stimulus combinations clearly different and inhibitory at suprathreshold would, at threshold, be detectable at a level equivalent to probability summation. Some suitable stimulus combinations could include rectangles varying in orientation and circular targets varying in area. An exploration of binocular summation with these kinds of stimuli might serve to define empirically the rules used by the binocular visual system to classify the equivalence of input at the level of forced-choice detectability.

The main conclusion supported by the present results is that different stimuli, as defined by positive and negative flashes, are detected as though they were separate and independent events, even when simultaneously presented. The data provide no support for either the energy integration or inhibition hypotheses initially entertained, and both can be rejected, at least for the case of positive and negative flashes. 


\section{REFERENCES}

Blake, R., \& Fox, R. The psychophysical inquiry into binocular summation. Perception \& Psychophysics, 1973, 14, 161-185.

Eriksen, C. W. Independence of successive inputs and uncorrelated error in visual form perception. Journal of Experimental Psychology, 1966, 72, 26-35.

Eriksen, C. W., Greenspon, T. S., Lappin, J., \& Carlson, W. A. Binocular summation in the perception of form at brief durations. Perception \& Psychophysics, 1966, 1, 415-419.

Green, D. M., \& Swets, J. A. Signal detection theory and psychophysics. New York: Wiley, 1966.

Ikeda, M. Temporal summation of positive and negative flashes in the visual system. Journal of the Optical Society of America, 1965, 55, 1527-1534.

Matin, L. Binocular summation at the absolute threshold for peripheral vision. Journal of the Optical Society of America, $1962,52,1276-1286$.

Rashbass, C. The visibility of transient changes of luminance. Journal of Physiology, 1970, 210, 165-186.

Swets, J. A. (Ed.) Signal detection and recognition by human observers: Contemporary readings. New York: Wiley, 1964.

Thomas, J. P., \& Kerr, L. G. Evidence of role of size-tuned mechanisms in increment threshold task. Vision Research, $1971,11,647-655$.

Westendorf, D. H., Blake, R. R., \& Fox, R. Binocular summation of equal-energy flashes of unequal duration. Perception \& Psychophysics, 1972, 12, 445-448.

\section{NOTES}

1. Predictions of binocular performance from monocular must assume independence of sensitivity variation in each eye as Matin (1962) has discussed in detail. Two major sources of dependence are variations within blocks and across sessions. To estimate dependence, correlations between monocular performance within blocks and across sessions were computed for each S. In all cases, the correlations were small and insignificant, thereby justifying the assumption of independence. A similar outcome was found for the data of Westendorf et al (1972), but not reported in that paper. It is likely that the use of a randomized block design operates to minimize correlation.

2. The general form of the Eriksen model, described in Eriksen (1966), is a multistate model whereby variations in perceptual sensitivity of the Os are indexed by confidence-rating scales, such scales being used to approximately weight corresponding hit rates. In this case, where rating-scale information is not available, the model reduces to two states, a hit state and a guess state. The reduction to two states ignores information about sensitivity variations and tends to overpredict the anticipated performance level, as discussed in detail by Eriksen (1966) and Eriksen et al (1966). But, as Eriksen et al note, the effect of the overprediction is small, on the order of $3 \%-5 \%$, a result similar to that found by Westendorf, Blake, and Fox (1972). Application of the two-state version of the Eriksen model yielded similar results in the present experiment.

For a three-choice task, the two-state model predicts:

$$
P_{B}=\left(1-G_{L} G_{R}\right)+1 / 3 G_{R} G_{L}
$$

where $G_{R}=3 / 2\left(1-P_{R}\right)$ and $G_{L}=3 / 2\left(1-P_{L}\right)$.

$P_{R}, P_{L}$, and $P_{B}$ are the probabilities of detection with the right eye, left eye, and both eyes, respectively. $G_{R}$ and $G_{L}$ are the estimated percentage of guess trials (both correct and incorrect) for the right and left eyes, respectively.

3. The integration model described explicitly by Green and Swets (1966) consists simply of obtaining a combined d' value from the square root of the sum of the squares of the $d^{\prime}$ values for each eye:

$$
d_{b}^{\prime}=\sqrt{d_{1}^{\prime 2}+d_{r}^{\prime 2}}
$$

where $d^{\prime}{ }_{b}=d^{\prime}$ in the binocular case, $d^{\prime}{ }_{1}=$ the $d^{\prime}$ obtained for the left eye alone, and $d^{\prime}{ }_{r}=d^{\prime}$ obtained for the right eye alone. Estimates of $d^{\prime}{ }_{1}$ and $d^{\prime}{ }_{r}$ were made from the mean monocular hit rates for the left eye and right eye averaged across sessions. Conversion of hit rates to $d^{\prime}$ for a three-alternative forced-choice condition was performed by a graphic method based upon Elliott (see Appendix 1, by Elliott, in Swets, 1964).

One referee commented that since $d^{\prime}$ is not linear with different hit rates, it may be inappropriate to estimate d' from pooled data, especially in cases where appreciable interesession variability is present. Intrigued by this suggestion, we estimated $d^{\prime}$ by taking the $d^{\prime}$ of each session and securing a mean $d^{\prime}$ value. Applying $d^{\prime}$ values obtained in this manner to the integration model yielded predicted performance that was, in all cases, within $1 \%$ of the values obtained using pooled hit rates.

(Received for publication June 8, 1973; revision received August 6, 1973.) 